\title{
Graph Based Transforms based on Graph Neural Networks for Predictive Transform Coding
}

\author{
Debaleena Roy, Tanaya Guha, and Victor Sanchez \\ Department of Computer Science, University of Warwick, Coventry, UK \\ \{debaleena.roy, tanaya.guha,v.f.sanchez-silva\}@warwick.ac.uk
}

In recent days, Graph Based Transforms (GBTs) have shown promising results [1] for data decorrelation and energy compaction for block-based predictive transform coding (PTC) using intra-prediction. This work introduces the GBT-NN, a novel class of Graph-based Transform based on neural network within the context of blockbased PTC using intra-prediction. The GBT-NN aims to perform as well as the KLT without requiring to explicitly compute the covariance matrix for each residual block to be transformed. The predicted residual is used to compute the all-connected adjacency matrix to be used as the input for the trained GBT-NN, which predicts the all-connected adjacency matrix of the current block (see Fig. 1 (a)).

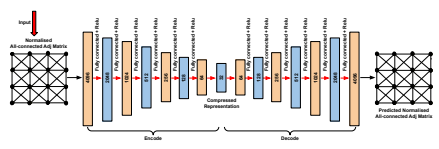

(a)

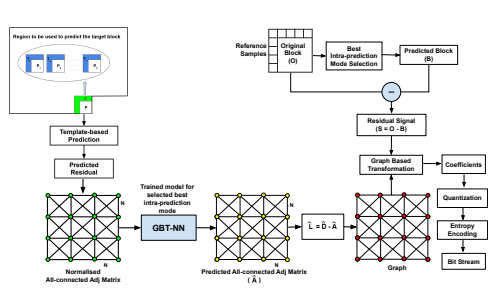

(b)

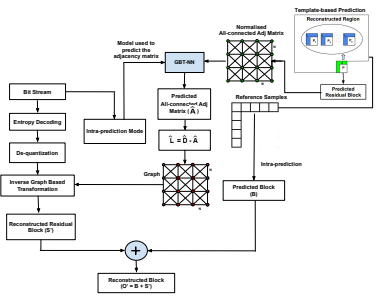

(c)

Figure 1: (a) Architecture of the proposed GBT-NN for the case of $8 \times 8$ blocks. (b) Block diagram of the proposed framework for encoding. (c) Block diagram for decoding.

Our framework for coding/decoding is depicted in Fig. 1. Note that the decoder needs information to reconstruct the graph which may lead to an increased overhead. Our framework adopts a template-based prediction strategy to predict residuals to avoid such overhead. Our evaluations on 40 different gray level frames of class A, B, C, D, E and SC and 10 pathology images show that the GBT-NN outperforms the DST, DCT by $10.46 \%, 6.37 \%$ in terms of preserved energy (PE) if only $5 \%$ of the largest coefficients are used to reconstruct the signal (Table 1).

Table 1: Average PE (in \%) and MSE using a small percentage of the largest coefficients.

\begin{tabular}{|l|cc|cc|cc|}
\cline { 2 - 7 } \multicolumn{1}{c|}{} & \multicolumn{4}{c|}{ Percentage of coefficients used } \\
\cline { 2 - 7 } \multicolumn{1}{c|}{} & \multicolumn{2}{c|}{$1 \%$} & \multicolumn{2}{c|}{$5 \%$} & \multicolumn{2}{c|}{$10 \%$} \\
& PE & MSE & PE & MSE & PE & MSE \\
\hline DCT & 16.84 & 82.99 & 52.11 & 48.38 & 69.07 & 32.03 \\
DST & 16.14 & 83.69 & 50.18 & 50.64 & 66.88 & 34.24 \\
GBT-NN & $\mathbf{1 8 . 9 7}$ & $\mathbf{7 8 . 7 2}$ & $\mathbf{5 5 . 4 3}$ & $\mathbf{4 4 . 4 6}$ & $\mathbf{7 2 . 4 0}$ & $\mathbf{2 8 . 9 4}$ \\
\hline
\end{tabular}

References

[1] D. I. Shuman, S. K. Narang, P. Frossard, A. Ortega, and P. Vandergheynst, "The emerging field of signal processing on graphs: Extending high-dimensional data analysis to networks and other irregular domains," IEEE Signal Processing Magazine, vol. 30, no. 3, pp. 83-98, 2013. 